EPJ Web of Conferences 71, 00073 (2014)

DOI: 10.1051/epjconf/20147100073

(C) Owned by the authors, published by EDP Sciences, 2014

\title{
Hadron yield ratios in an effective relativistic equation of state
}

\author{
A. Lavagno \\ ${ }^{1}$ Department of Applied Science and Technology, Politecnico di Torino, I-10129 Torino, Italy \\ ${ }^{2}$ Istituto Nazionale di Fisica Nucleare (INFN), Sezione di Torino, I-10126 Torino, Italy
}

\begin{abstract}
We investigate an effective relativistic equation of state at finite values of temperature and baryon chemical potential with the inclusion of the full octet of baryons, the Delta-isobars and the lightest pseudoscalar and vector meson degrees of freedom. These last particles have been introduced within a phenomenological approach by taking into account of an effective chemical potential and mass depending on the self-consistent interaction between baryons. In this framework, we study of the hadron yield ratios measured in central heavy ion collisions over a broad energy range.
\end{abstract}

\section{Introduction}

It is well known that mesons play a crucial role in the hadronic Equation of State (EOS) especially in the regime of low baryon density and high temperature. Such particles are strongly interacting in the environment with the other particles. In this contribution, we are going to study an effective relativistic mean field model with the inclusion of the full octet of baryons, the $\Delta$-isobars and the lightest pseudoscalar and vector mesons, by requiring the global conservation of baryon number, electric charge fraction and zero net strangeness. The meson degrees of freedom are incorporated in the EOS as a quasi-particle Bose gas with an effective meson chemical potential $\mu^{*}$ and an effective mass $m^{*}$ expressed in terms of the $\sigma, \omega, \rho$ meson fields, responsible for the self-consistent mean field interaction [1]. In this framework the hadron yield ratios have been studied and compared with the experimental ratios measured in central heavy ion collisions from AGS to RHIC energy range.

\section{The effective relativistic mean field model}

The total Lagrangian density $\mathcal{L}$ can be written as

$$
\mathcal{L}=\mathcal{L}_{\text {octet }}+\mathcal{L}_{\Delta}+\mathcal{L}_{\mathrm{qpm}}
$$

where $\mathcal{L}_{\text {octet }}$ stands for the full octet of the lightest baryons $\left(p, n, \Lambda, \Sigma^{+}, \Sigma^{0}, \Sigma^{-}, \Xi^{0}, \Xi^{-}\right)$interacting with $\sigma, \omega, \rho$ meson fields; $\mathcal{L}_{\Delta}$ corresponds to the degrees of freedom for the $\Delta$-isobars $\left(\Delta^{++}, \Delta^{+}, \Delta^{0}\right.$, $\Delta^{-}$) and $\mathcal{L}_{\text {qpm }}$ is related to a quasi-particle gas of the lightest pseudoscalar and vector mesons with effective chemical potentials and masses (see below for details).

This is an Open Access article distributed under the terms of the Creative Commons Attribution License 2.0, which permits unrestricted use, distribution, and reproduction in any medium, provided the original work is properly cited. 
The Lagrangian for the self-interacting octet of baryons can be written as [2]

$$
\begin{aligned}
\mathcal{L}_{\mathrm{octet}}= & \sum_{k} \bar{\psi}_{k}\left[i \gamma_{\mu} \partial^{\mu}-\left(M_{k}-g_{\sigma k} \sigma\right)-g_{\omega k} \gamma_{\mu} \omega^{\mu}-g_{\rho k} \gamma_{\mu} \vec{t} \cdot \vec{\rho}^{\mu}\right] \psi_{k}+\frac{1}{2}\left(\partial_{\mu} \sigma \partial^{\mu} \sigma-m_{\sigma}^{2} \sigma^{2}\right) \\
& -\frac{1}{3} a\left(g_{\sigma N} \sigma\right)^{3}-\frac{1}{4} b\left(g_{\sigma N} \sigma^{4}\right)+\frac{1}{2} m_{\omega}^{2} \omega_{\mu} \omega^{\mu}+\frac{1}{4} c\left(g_{\omega N}^{2} \omega_{\mu} \omega^{\mu}\right)^{2}+\frac{1}{2} m_{\rho}^{2} \vec{\rho}_{\mu} \cdot \vec{\rho}^{\mu} \\
& -\frac{1}{4} F_{\mu \nu} F^{\mu \nu}-\frac{1}{4} \vec{G}_{\mu \nu} \vec{G}^{\mu \nu}
\end{aligned}
$$

where the sum runs over the full octet of baryons, $M_{k}$ is the vacuum baryon mass of index $k$, the quantity $\vec{t}$ denotes the isospin operator that acts on the baryon.

The Lagrangian density concerning the $\Delta$-isobars can then be expressed as [3-5]

$$
\mathcal{L}_{\Delta}=\bar{\psi}_{\Delta v}\left[i \gamma_{\mu} \partial^{\mu}-\left(M_{\Delta}-g_{\sigma \Delta} \sigma\right)-g_{\omega \Delta} \gamma_{\mu} \omega^{\mu}\right] \psi_{\Delta}^{\nu},
$$

where $\psi_{\Delta}^{v}$ is the Rarita-Schwinger spinor for the $\Delta$-baryon. Due to the uncertainty on the meson- $\Delta$ coupling constants, we limit ourselves to consider only the coupling with $\sigma\left(g_{\sigma \Delta}\right)$ and $\omega\left(g_{\omega \Delta}\right)$ meson fields, more of which are explored in the literature [4-6].

In the RMF model the interaction gives an effective mass of $i$-th baryon which depends on the $\sigma$ meson fields by means the relation $M_{i}^{*}=M_{i}-g_{\sigma i} \sigma$. In the meson-field equations appear the $\rho_{i}^{B}$ and $\rho_{i}^{S}$ are the baryon density and the scalar density of the particle of index $i$, respectively. They are given by

$$
\begin{aligned}
& \rho_{i}^{B}=\gamma_{i} \int \frac{\mathrm{d}^{3} k}{(2 \pi)^{3}}\left[f_{i}(k)-\bar{f}_{i}(k)\right], \\
& \rho_{i}^{S}=\gamma_{i} \int \frac{\mathrm{d}^{3} k}{(2 \pi)^{3}} \frac{M_{i}^{*}}{E_{i}^{*}}\left[f_{i}(k)+\bar{f}_{i}(k)\right],
\end{aligned}
$$

where $\gamma_{i}=2 J_{i}+1$ is the degeneracy spin factor of the $i$-th baryon $\left(\gamma_{\text {octet }}=2\right.$ for the baryon octet and $\left.\gamma_{\Delta}=4\right)$ and $f_{i}(k)$ and $\bar{f}_{i}(k)$ are the fermion particle and antiparticle distributions

$$
\begin{aligned}
f_{i}(k) & =\frac{1}{\exp \left\{\left(E_{i}^{*}(k)-\mu_{i}^{*}\right) / T\right\}+1}, \\
\bar{f}_{i}(k) & =\frac{1}{\exp \left\{\left(E_{i}^{*}(k)+\mu_{i}^{*}\right) / T\right\}+1} .
\end{aligned}
$$

The effective chemical potentials $\mu_{i}^{*}$ are given in terms of the chemical potentials $\mu_{i}$ by means of the following relation

$$
\mu_{i}^{*}=\mu_{i}-g_{\omega i} \omega-g_{\rho i} t_{3 i} \rho,
$$

where $t_{3 i}$ is the third component of the isospin of $i$-th baryon. The baryon effective energy is defined as $E_{i}^{*}(k)=\sqrt{k^{2}+M_{i}^{* 2}}$.

Because we are going to describe the nuclear EOS at finite temperature and density with respect to strong interaction, we have to require the conservation of three "charges": baryon number (B), electric charge (C) and strangeness number (S) [7, 8]. Each conserved charge has a conjugated chemical potential and the system is described by three independent chemical potentials: $\mu_{B}, \mu_{C}$ and $\mu_{S}$. Therefore, the chemical potential of particle of index $i$ can be written as

$$
\mu_{i}=b_{i} \mu_{B}+c_{i} \mu_{C}+s_{i} \mu_{S},
$$

where $b_{i}, c_{i}$ and $s_{i}$ are, respectively, the baryon, the electric charge and the strangeness quantum numbers of $i$-th hadronic species. All the aforementioned equations must be evaluated self-consistently 
fulfilling the chemical equilibrium and by imposing the constraints of baryon number, electric charge and strangeness. Therefore, at a given temperature $T$, baryon density $\rho_{B}$, net electric charge fraction $Z / A\left(\rho_{C}=Z / A \rho_{B}\right)$ and zero net strangeness of the system $\left(\rho_{S}=0\right)$, the chemical potentials $\mu_{B}, \mu_{C}$ and $\mu_{S}$ are univocally determined by the following equations

$$
\begin{aligned}
& \rho_{B}=\sum_{i} b_{i} \rho_{i}\left(T, \mu_{B}, \mu_{C}, \mu_{S}\right), \\
& \rho_{C}=\sum_{i} c_{i} \rho_{i}\left(T, \mu_{B}, \mu_{C}, \mu_{S}\right), \\
& \rho_{S}=\sum_{i} s_{i} \rho_{i}\left(T, \mu_{B}, \mu_{C}, \mu_{S}\right),
\end{aligned}
$$

where the sums run over all considered particles (baryons and mesons).

The numerical evaluation of the above quantities can be performed if the meson-nucleon, $-\Delta$ and -hyperon coupling constants are known. Concerning the nucleon coupling constants $\left(g_{\sigma N}, g_{\omega N}, g_{\rho N}\right)$, they are determined to reproduce the bulk properties of equilibrium nuclear matter such as the saturation densities, the binding energy, the symmetric energy coefficient, the compression modulus and the effective Dirac mass at saturation. In this investigation we consider two different parameter sets: the set marked TM1, from Ref.[9], and GM3, from Glendenning and Moszkowski [10]. The above EOSs are compatible with intermediate heavy ion collisions constraints and extensively used in various high density astrophysical applications [11-16].

As regards the implementation of hyperon degrees of freedom, they come from determination of the corresponding meson-hyperon coupling constants that have been fitted to hypernuclear properties [17]. The adopted coupling constants and the vacuum masses are the same as in Ref. [11].

Concerning the formation of $\Delta$-isobar matter at finite temperature and density, if the SU(6) symmetry is exact, one can adopt the universal couplings $x_{\sigma \Delta}=g_{\sigma \Delta} / g_{\sigma N}=1$ and $x_{\omega \Delta}=g_{\omega \Delta} / g_{\omega N}=1$. However, the SU(6) symmetry is not exactly fulfilled and one may assume the scalar coupling ratio $x_{\sigma \Delta}>1$ with a value close to the mass ratio of the $\Delta$ and the nucleon [5]. On the other hand, QCD finite-density sum rule results show that the Lorentz vector self-energy for the $\Delta$ is significantly smaller than the nucleon vector self-energy implying therefore $x_{\omega \Delta}<1$ [6].

Without loss of generality, we can limit our investigation to move only in a side of a triangle region by fixing $x_{\omega \Delta}=1$ and varying $x_{\sigma \Delta}$ from unity to a maximum value compatible with the physical conditions mentioned in Ref. [5]. Comparable conclusions are obtained with any other compatible choice of the two coupling ratios (see Ref. [11] for a more detailed discussion about the value of the $\Delta$-couplings and the formation of $\Delta$ metastable matter for different EOSs). In this investigation, we fix the scalar coupling ratio to the value $x_{\sigma \Delta}=1.25$ in the TM1 and $x_{\sigma \Delta}=1.40$ in the GM3 parameter set, for which the $\Delta$ metastable condition is not realized. In addition, we consider the case of $x_{\sigma \Delta}=1.33$ in the TM1 and $x_{\sigma \Delta}=1.50$ in the GM3 parameter set, corresponding to the maximum value in which $\Delta$ metastable state can be realized [11]. Therefore, we consider two very different scenarios (not metastable and metastable $\Delta$-matter) for the two different parameter sets (note that, when a metastable state is not realized, decays rate are not taken into account in this approach).

It is well known that the lightest pseudoscalar and vector mesons play a crucial role in the EOS, especially at low baryon density and high temperature. On the other hand, the contribution of the $\pi$ mesons (and other pseudoscalar and pseudovector fields) vanishes at the mean-field level. From a phenomenological point of view, we can take into account the meson particle degrees of freedom by adding their one-body contribution to the thermodynamical potential, that is, the contribution of a quasi-particle Bose gas with an effective chemical potential $\mu_{j}^{*}$ and an effective mass $m_{j}^{*}$ for the 
$j$-meson, which contain the self-consistent interaction of the meson fields. The details of the this phenomenological model are discussed in Ref. [1].

\section{Results and discussion}

We are going to compare the hadronic ratios, obtained in the framework of the effective EOS, with the yield ratios measured in central high energy heavy ion collisions from AGS to RHIC energy range. At this scope, as in statistical thermal models, we consider the temperature and the baryon chemical potential as free parameters of the hadronic EOS (we do not consider proper volume corrections and strangeness suppression factor) and we adjust them by fitting the data minimizing the $\chi^{2}$ distribution [18-21]

$$
\chi^{2}=\sum_{i}\left(\frac{R_{i}^{\exp }-R_{i}^{\mathrm{eos}}}{\sigma_{i}}\right)^{2},
$$

where $R_{i}^{\exp }$ is the measure of the $i$ th ratio yields with its relative uncertainty $\sigma_{i}$ (sum in quadrature of statistical and systematic experimental errors) and $R_{i}^{\text {eos }}$ is the corresponding value obtained from the effective hadronic EOS.

It is obvious that the chemical freeze-out cannot correctly be described as a mixture of the lightest baryons and mesons considered in the above EOS without properly considering decays, rescattering, annihilation effects and, eventually, nonequilibrium processes. Furthermore, in the considered EOS the particles are off-shell (with an effective mass) and, in the determination of the hadronic ratios, the effects of any mechanism to bring them on-shell are not taken into account. Therefore, the obtained temperatures and baryon chemical potentials cannot properly be considered as the freeze-out parameters. However, as in Refs. [22, 23], such a fitting procedure can give interesting indications on the nature of the in-medium nuclear interaction at finite temperature and density regime.

Taking into account the above observations, we limit ourselves to study only hadron yield ratios (therefore the enclosed volume of the system plays no role in the analysis) and, for simplicity, to consider a limited set of experimental ratios. In order to make such a choice as little as possible arbitrary, we include in the fit, for SPS and RHIC data, the same five independent yield ratios considered in the recent data analysis of Refs. [24, 25]: $\pi^{-} / \pi^{+}, K^{-} / K^{+}, \bar{p} / p, K^{-} / \pi^{-}, \bar{p} / \pi^{-}$and, at the AGS energies, we choose the most similar set of data on the basis of the experimental ratios considered in Ref. [19]. Therefore, except in the case of beam energy $\sqrt{s_{N N}}=130 \mathrm{GeV}$ (see below for details), we refer to [19] and references therein for the AGS and SPS midrapidity data and to [24] for the RHIC midrapidity data. In the central Au-Au collisions at AGS and RHIC, the electric charge fraction has been fixed to $Z / A=0.401$, while in the SPS $\mathrm{Pb}-\mathrm{Pb}$ collisions $Z / A=0.394$. As already stated, we require a zero net strangeness at all energies, therefore, at this stage the analysis does not consider the possible presence of a non-vanishing net strangeness at midrapidity.

In Table 1, for the TM1 parameter set and with $x_{\sigma \Delta}=1.25$, we summarize the complete results for the best fits reporting, for any considered beam energy, the obtained free parameters $T$ and $\mu_{B}$ with the corresponding $\chi_{\text {dof }}^{2}=\chi^{2} / N_{\text {dof }}$ (where $N_{\text {dof }}=2$ at $\sqrt{s_{N N}}=2.7 \mathrm{GeV}$ and $N_{\text {dof }}=3$ in the other cases). For comparison, in the round brackets are reported the results without considering effective meson chemical potentials and masses (free meson gas), while in the square bracket are given the values of $\chi_{\text {dof }}^{2}$ obtained with effective meson chemical potentials only. Moreover, in the last line of the Tables is reported the best fit at the beam energy $\sqrt{s_{N N}}=130 \mathrm{GeV}$ corresponding to the same nine particle ratios and experimental data considered in Refs. [18, 22] $\left(N_{\text {dof }}=7\right)$, and analyzed in Ref. [23].

As expected the obtained values of $\chi_{\mathrm{dof}}^{2}$ are very high without considering effective meson masses (except for the case $\sqrt{s_{N N}}=2.7 \mathrm{GeV}$ for which a very low fraction of mesons takes place). Comparable high values of $\chi_{\text {dof }}^{2}$ are also obtained by considering effective meson chemical potentials only. 
Table 1. Summary of the results of the fits using the particle ratios extracted from Refs. [19, 24] (see text for details) for the TM1 parameter set. For comparison, in the round brackets are reported the respective values obtained without effective meson chemical potentials and masses (free gas of mesons). In the square brackets are given the values of $\chi_{\text {dof }}^{2}$ obtained with effective meson chemical potentials only (no effective masses). In the last line, the asterisk means that the fits are related to the same nine particle ratios and experimental data considered in Refs. [18, 22, 23].

\begin{tabular}{c|ccc}
\hline \hline \multirow{2}{*}{$\begin{array}{c}\sqrt[3]{s_{N N}} \\
(\mathrm{GeV})\end{array}$} & \multicolumn{3}{|c}{$x_{\sigma \Delta}=1.25$} \\
\cline { 2 - 4 } & $T(\mathrm{MeV})$ & $\mu_{B}(\mathrm{MeV})$ & $\chi_{\text {dof }}^{2}$ \\
\hline 2.70 & $51(49)$ & $743(741)$ & $1.19(1.09)[1.11]$ \\
3.32 & $83(68)$ & $656(666)$ & $1.38(2.43)[2.73]$ \\
3.84 & $101(78)$ & $596(620)$ & $1.63(2.96)[3.04]$ \\
4.30 & $105(86)$ & $563(589)$ & $3.11(2.76)[2.88]$ \\
4.85 & $107(106)$ & $534(551)$ & $1.90(11.34)[11.24]$ \\
8.76 & $125(121)$ & $405(500)$ & $2.66(7.87)[8.02]$ \\
12.3 & $133(128)$ & $346(456)$ & $2.98(16.64)[16.82]$ \\
17.3 & $137(131)$ & $306(431)$ & $1.66(13.61)[12.97]$ \\
62.4 & $153(123)$ & $97(49)$ & $1.67(17.66)[17.71]$ \\
130 & $154(128)$ & $46(23)$ & $1.27(15.61)[15.60]$ \\
200 & $154(146)$ & $34(24)$ & $0.40(9.57)[9.52]$ \\
\hline $130^{*}$ & $154(149)[149]$ & $48(33)$ & $0.56(4.22)[4.21]$ \\
\hline \hline
\end{tabular}

On the other hand, we can see that the introduction of the effective meson masses play a crucial role with results in very good agreement for both EOSs.

From Table 1, the energy dependence of the two free parameters, $\mu_{B}$ and $T$, exhibits a monotonic dependence on the collision energy and appears very similar to the statistical thermal model results where $\mu_{B}$ decreases all the way up to RHIC energies, while $T$ rises rather sharply up to the lower SPS energies, $\sqrt{s_{N N}} \approx 10 \mathrm{GeV}$, and reaches afterwards almost constant values at RHIC energies.

In Fig. 1, we report a parametrization of the energy dependence of baryon chemical potential and temperature obtained from our best fits for the TM1 set (dashed lines) with $x_{\sigma \Delta}=1.33$ and for the GM3 set with $x_{\sigma \Delta}=1.40$ (solid lines); the other $\Delta$-coupling cases are not reported because they are very strictly to the previous ones. The energy dependence of the two free parameters, $\mu_{B}$ and $T$, exhibits a monotonic dependence on the collision energy and appears very similar to the statistical thermal model results where $\mu_{B}$ decreases all the way up to RHIC energies, while $T$ rises rather sharply up to the lower SPS energies, $\sqrt{s_{N N}} \approx 10 \mathrm{GeV}$, and reaches afterwards almost constant values at RHIC energies.

In this context, it is proper to remember that the values of temperature and baryon chemical potential, obtained from the fitting procedure with the experimental data, cannot properly be considered as the freeze-out parameters without taking into account particle decays, rescattering and annihilation effects. On the other hand, a correct reproduction of all particle production yields lies outside the scope of this paper. The main purpose of such a phenomenological comparison with experimental data is to provide useful indications about the relevance of the meson-baryon and meson-meson interaction in regime of finite values of temperature and baryon chemical potential where meson degrees of freedom become progressively more important. 

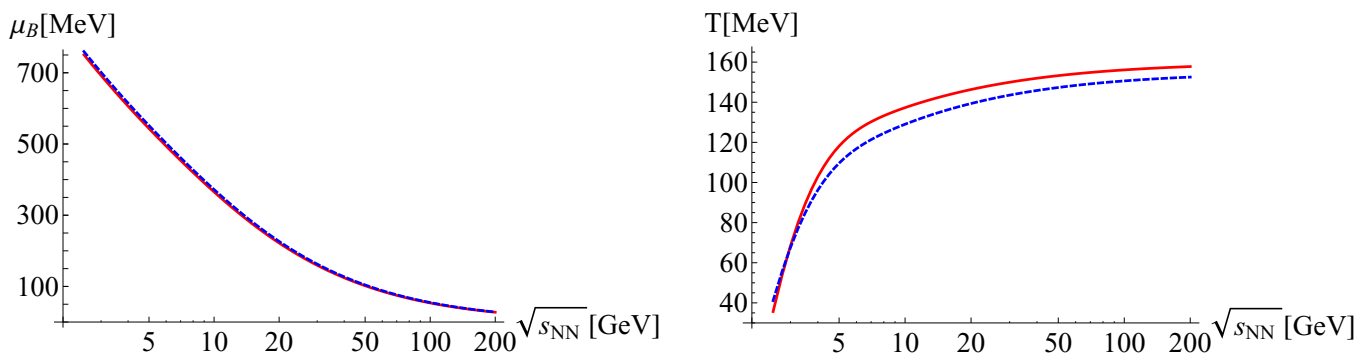

Figure 1. The energy dependence of baryon chemical potential (upper panel) and temperature (lower panel). The lines are the parameterizations for $\mu_{B}$ and $T$ obtained from fits with different particle ratios (see text for details). The dashed lines correspond to the TM1 parameter set $\left(x_{\sigma \Delta}=1.33\right)$ and the solid lines correspond to the GM3 parameter set $\left(x_{\sigma \Delta}=1.40\right)$.

Using the obtained values of $\mu_{B}$ and $T$ at different beam energies, by means the EOS, it is possible to obtain other relevant thermodynamic variables and to calculate the energy dependence of various hadron ratios. In Fig. 2, we report the energy dependence of antiparticle to particle ratios (we refer to [19] and reference therein for the experimental data reported in the figure). Calculations reproduce rather well all these experimental ratios with a small variation for the two different adopted TM1 and GM3 model and for different values of $x_{\sigma \Delta}$.

In conclusion, we have found that the presence of $\Delta$-isobar degrees of freedom play a crucial role in our scheme, in particular we have seen that a better agreement with the experimental data can be reached with lower values of the coupling ratio $x_{\sigma \Delta}$ (corresponding to a not metastable $\Delta$-isobar state) at lower beam energies and with higher values of $x_{\sigma \Delta}$ (corresponding to the formation of a $\Delta$-isobar metastable state) at higher beam energies. From a phenomenological point of view, the coupling ratio $x_{\sigma \Delta}$ could be interpreted as a parameter which takes into account in an effective manner the formation of resonance states, very relevant in regime of high temperatures and low baryon chemical potentials. 

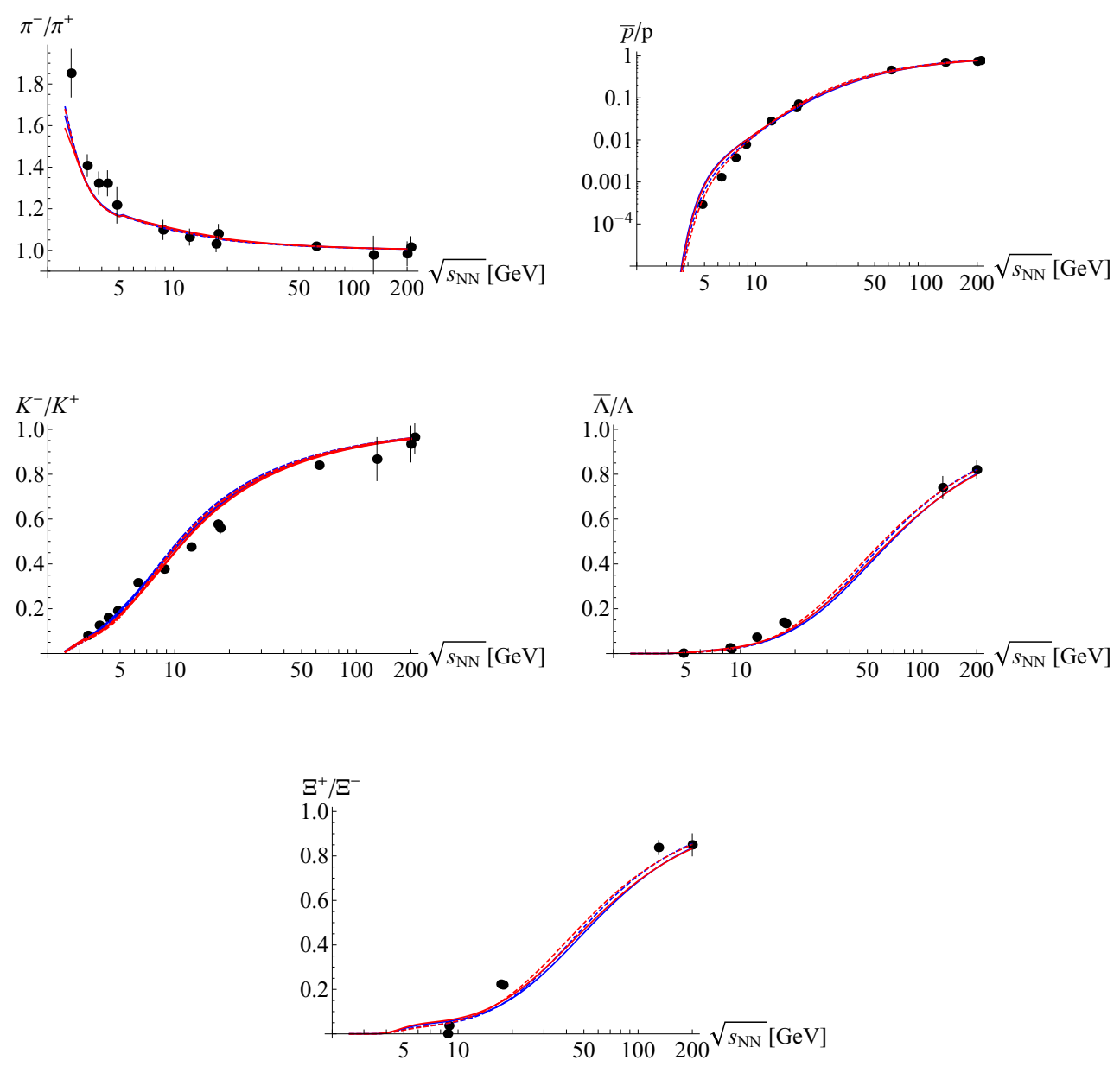

Figure 2. The energy dependence of antiparticle/particle ratios for different parameterizations in the TM1 $\left(x_{\sigma \Delta}=\right.$ 1.25 and $\left.x_{\sigma \Delta}=1.33\right)$ and GM3 $\left(x_{\sigma \Delta}=1.4\right.$ and $\left.x_{\sigma \Delta}=1.5\right)$ models.

\section{References}

[1] A. Lavagno, Eur. Phys. J. A 49, 102 (2013).

[2] N.K. Glendenning, Phys. Lett. B 114, 392 (1982).

[3] J. Boguta, Phys. Lett. B 109, 251 (1982).

[4] Z. Li, G. Mao, Y. Zhuo, W. Greiner, Phys. Rev. C 56, 1570 (1997).

[5] D.S. Kosov, C. Fuchs, B.V. Martemyanov, A. Faessler, Phys. Lett. B 421, 37 (1998).

[6] X. Jin, Phys. Rev. C 51, 2260 (1995). 
[7] A. Lavagno, D. Pigato, Phys. Rev. C 86, 024917 (2012).

[8] A. Lavagno, D. Pigato, J. Phys. G: Nucl. Part. Phys. 39, 125106 (2012).

[9] Y. Suguhara, H. Toki, Nucl. Phys. A 579, 557 (1994).

[10] N.K. Glendenning, S.A. Moszkowski, Phys. Rev. Lett. 67, 2414 (1991).

[11] A. Lavagno, Phys. Rev. C 81, 044909 (2010).

[12] P. Danielewicz, R. Lacey, and W. G. Lynch, Science 298, 1592 (2002).

[13] M. Di Toro et al., Nucl. Phys. A 775, 102 (2006).

[14] L. Bonanno, A. Drago, A. Lavagno, Phys. Rev. Lett. 99, 242301 (2007).

[15] W.M. Alberico, A. Lavagno, Eur. Phys. J. A 40, 313 (2009).

[16] A. Lavagno, D. Pigato, Eur. Phys. J. A 47, 52 (2011).

[17] J. Schaffner, C.B. Dover, A. Gal, C. Greiner, H. Stöcker, Phys. Rev. Lett. 71, 1328 (1993).

[18] P. Braun-Munzinger, D. Magestro, K. Redlich, J. Stachel, Phys. Lett. B 518, 41 (2001).

[19] A. Andronic, P. Braun-Munzinger, J. Stachel, Nucl. Phys. A 772, 167 (2006).

[20] F. Becattini, J. Manninen, M. Gaździcki, Phys. Rev. C 73, 044905 (2006).

[21] J. Cleymans, H. Oeschler, K. Redlich, S. Wheaton, Phys. Rev. C 73, 034905 (2006).

[22] D. Zschiesche et al., Phys. Lett. B 547, 7 (2002).

[23] M. Chiapparini et al., Nucl. Phys. A 826, 178 (2009).

[24] B.I. Abelev et al. (STAR Collab.), Phys. Rev. C 79, 034909 (2009) and reference therein.

[25] B.I. Abelev et al. (STAR Collab.), Phys. Rev. C 81, 024911 (2010). 